Ann. Biol. anim. Bioch. Biophys, I970, $10(\mathrm{I})$, I65-170.

\title{
FRACTIONNEMENT DES ACIDES RIBONUCLÉIQUES DE TRANSFERT DE MAMMIFÈRES SUR BENZOYL-DIÉTHYLAMINOÉTHYLCELLULOSE
}

\author{
G. PÉTRISSANT \\ avec Ia collaboration technique de Monique Boisnard et Claudine Puissant \\ Laboratoire de Physiologie de la Lactation, \\ Centre national de Recherches zootechniques, 78 -Jouy-en-Josas \\ Institut national de la Recherche agronomique
}

RÉSUMÉ

Au cours d'une étude systématique des techniques de fractionnement des acides ribonucléiques de transfert chez les Mammifères la benzoyl-diéthylaminoéthylcellulose a été utilisée pour la séparation des acides ribonucléiques de foie de Lapin et de glande mammaire de Brebis.

On obtient par cette méthode une répartition des acides ribonucléiques étudiés en deux groupes principaux. Les profils obtenus à partir des deux organes sont identiques.

Il existe également des ressemblances intéressantes avec les acides ribonucléiques de levure. On constate cependant des différences quant au nombre et à la proportion relative des divers iso-accepteurs.

Les résultats obtenus montrent que la technique utilisée peut être appliquée à la purification des $t$-ARN (I) méthionine et sérine.

\section{INTRODUC'TION}

L'étude du contrôle endocrinien de la glande mammaire par les hormones antéhypopkysaires nous a conduit à formuler l'hypothèse d'une participation des $t$-ARN à la régulation de la synthèse protéique dans cet organe. Nous avons décrit une

(1) Abréviations

$t$-ARN : acide ribonucléique de transfert

RPC : reversed phase chromatography

MAK : methyl-albumin kieselguhr

DEAE-Sephadex : diéthylaminoéthyl-Sephadex

BD-cellulose : benzoyldiéthylaminoéthyl-cellulose

GSH : glutathion réduit 
méthode de préparation et quelques propriétés physico-chimiques et biologiques de cette classe de macromolécules (PÉTRISSANT, I968-I969). La purification de certains $t$-ARN spécifiques constitue l'étape suivante de notre programme. Dans ce but, nous avons étudié le fractionnement des $t$-ARN de foie de Lapin et de glande mammaire de Brebis par chromatographie en phase inversée (RPC) ainsi que sur colonne de MAK, de DEAE-Sephadex et de BD-cellulose. Le présent article expose les résultats obtenus grâce à cette dernière technique dans le cas des $t$-ARN alanine, arginine, leucine, méthionine, sérine et valine.

\section{MATÉRIEL}

Les animaux utilisés pour la préparation du $t$-ARN et des amino-acyl-t-ARN-synthétases de foie sont des lapins mâles $N e r-Z$ ealand âgés de six mois environ.

Le $t$-ARN et les amino-acyl-t-ARN-synthétases de glande mammaire proviennent de brebis Préalpes sacrifiées entre le $20^{\mathrm{e}}$ et le $6 \mathrm{o}^{\mathrm{e}}$ jour de lactation.

La BD-cellulose est délivrée par Serva (Heidelberg). Les acides aminés radioactifs ${ }^{14} \mathrm{C}$ sont fournis par le C.E.A. (Saclay, France).

\section{MÉTHODES}

\section{Préparation des t- $A R N$}

Le $t$-ARN de foie et de glande mammaire est obtenu par la méthode de BRUNNGRABER (I962) avec les modifications suivantes : la purification sur DEAE-cellulose, réalisée par suspension de l'échangeur dans la solution d'acides nucléiques, est suivie d'une seconde extraction phénolique; elle est complétée par une précipitation sélective à l'isopropanol (DEUTScher, 1967) et par une incubation dans le Tris I,8 M, pH 8 (SARIN et ZameCNIK, 1964). Le rendement est de 50 mg de $t$-ARN brut pour roo g de tissu frais. La filtration sur gel de Sephadex G-Ioo montre que la préparation ne contient pas d'acides nucléiques de haut poids moléculaire et est contaminée par io p. Ioo environ d'ARN de type $5 \mathrm{~S}$.

\section{Préparation des amino-acyl-t-ARN-synthétases}

Ces enzymes sont obtenus par une adaptation (PÉTRISSANT, 1969) des techniques décrites par Muench et Berg (1966) pour la préparation des enzymes de E. coli.

\section{Chromatographie}

Les colonnes $(0,85 \times \mathrm{I} I 0 \mathrm{~cm})$ sont préparées selon Gillam et al. (I967). Toutes les solutions utilisées contiennent : acétate de sodium o, or $\mathrm{M}$, chlorure de $\mathrm{Mg}$ o, OI $\mathrm{M}$ et sont tamponnées à pH 4,5. Le t-ARN, $2000 \mathrm{DO}_{260}$ dans 6 à $8 \mathrm{ml} \mathrm{de} \mathrm{NaCl} 0,4 \mathrm{M}$, est adsorbé sur la colonne qui est ensuite lavée avec la même solution et éluée par un gradient linéaire de $\mathrm{NaCl}$ comme indiqué sur les figures I et 2.

\section{Localisation des $\mathrm{t}-A R N$ spécifiques}

Après lecture de la densité optique à $260 \mathrm{~m} \mu$, des échantillons de $50 \mu 1$ sont prélevés dans chaque fraction. A chacun d'eux sont ajoutés zo $\mu l$ de milieu d'incubation contenant: Tris $\mathrm{pH} 7,4$, I 5 micromoles, ATP r,35 micromole, $\mathrm{MgCl}_{2}$ I,8 micromole, GSH I,2 micromole, acide aminé radioactif $0, \mathrm{I}-0,2$ microcurie. Le volume final est ajusté à i $50 \mu l$ par addition d'eau et de ro $\mu l$ de préparation enzymatique (environ $200 \mu \mathrm{g}$ ). A la fin de l'incubation (2o minutes, $37^{\circ} \mathrm{C}$ ) le $t$-ARN 
est précipité par $2 \mathrm{ml}$ d'acide perchlorique $0,5 \mathrm{~N}$ en présence de DNA entraîneur. Les culots sont lavés à l'acide perchlorique et à l'alcool-éther et dissous dans l'acide formique concentré. La radioactivité est mesurée avec un compteur Tracerlab à fenêtre mince (efficacité : I 5 p. roo).

\section{RÉSULTATS}

L'examen des figures I et 2 permet de constater les faits suivants :

I. Le chromatogramme peut être divisé en trois fractions. La première (tubes 50-75) contient le $t$-ARN valine, la majeure partie du $t$-ARN alanine et l'un des $t$-ARN méthionine. La fraction centrale (tubes $75-\mathrm{IOO}$ ) renferme un pic hétérogène de $t$-ARN méthionine, un $t$-ARN sérine, la majeure partie des $t$-ARN arginine et leucine. La fraction terminale (tubes Ioo-I40) contient essentiellement un $t$-ARN sérine.

2. Tous les $t$-ARN étudiés sont hétérogènes. Les $t$-ARN leucine et arginine présentent un profil très complexe. La meilleure séparation des iso-accepteurs est obtenue dans le cas des $t$-ARN méthionine et sérine.

3. Il existe une excellente correspondance entre les $t$-ARN de foie de Lapin et de glande mammaire de Brebis quant au nombre et à la répartition des pics. La meilleure résolution constatée dans le cas de la glande mammaire est vraisemblablement due à l'étalement du gradient d'élution.

En utilisant les critères donnés par Hoskinson et KHORANa (1965), on contate que dans cette dernière expérience, le tube 57 contient du RNA méthionine à 42 p. 100 de pureté (706 $\mu \mu$ moles de méthionine par DO) et le tube I30 du RNA sérine à $27 \mathrm{p} .100$ de pureté (449 $\mu \mu$ moles de sérine par DO).

\section{DISCUSSION}

L'application à l'étude des $t$-ARN d'organismes supérieurs des techniques décrites par GiLLAM et al. (I967) pour la séparation des $t$-ARN de levure donne des résultats satisfaisants dont l'analyse conduit aux remarques suivantes :

I. La courbe de densité optique présentée par ces auteurs témoigne d'une finesse de fractionnement que nous n'avons pas atteinte. Les différences dans l'origine de l'échangeur utilisé, le $\mathrm{pH}$ du tampon d'élution et la nature du matériel analysé peuvent expliquer ce phénomène.

2. Les $t$-ARN alanine, letcine, méthionine et valine de levure et de Mammifères montrent de grandes analogies tant par leur emplacement respectif dans le diagramme d'élution que par leur hétérogénéité. Toutefois les proportions relatives des diverses sous-espèces peuvent être très différentes.

Le $t$-ARN sérine de Mammifères comme celui de levure présente un pic hétérogène élué très tardivement mais comprend également un pic très important confondu 

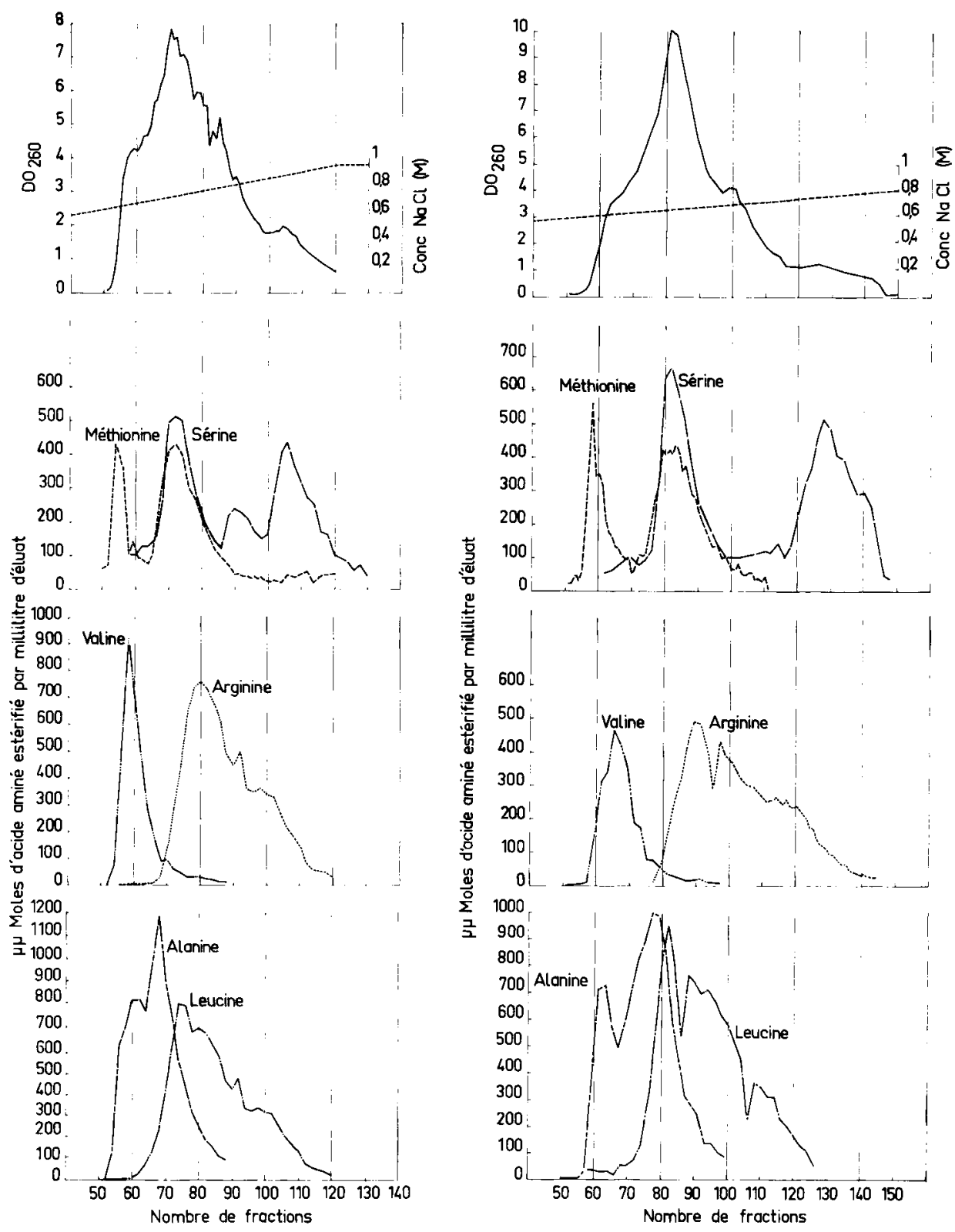

FIG. I. - Fractionnement de $\mathrm{t}-A R N$ de foie de Lapin sur $B D$-cellulose.

FIG. 2. - Fractionnement de $\mathrm{t}-A R N$ de glande mammaire de Brebis sur BD-cellulose.

Colonne de $0,85 \times$ r $10 \mathrm{~cm}$. Vitesse d'élution $24 \mathrm{ml} /$ heure. Température $25^{\circ} \mathrm{C}$. Volume des fractions $7,5 \mathrm{ml}$. L'activité acceptrice est mesurée comme décrit dans le texte après neutralisation de chaque fraction par Ioo $\mu l$ de Tris M. 
avec l'un des $t$-ARN méthionine, ainsi qu'un pic intermédiaire surtout apparent dans le foie.

Le $t$-ARN arginine, très étalé, semble plus hétérogène chez les Mammifères que dans la levure.

3. Les profils de radioactivité ci-dessus ont été obtenus en utilisant des enzymes homologues. Les résultats sont identiques lors de l'acylation des $t$-ARN de foie par des enzymes de glande mammaire et vice-versa.

4. Le pouvoir séparateur de la BD-cellulose est supérieur à celui de la MAK et du DEAE-Sephadex. Cet échangeur permet en particulier une meilleure séparation des iso-accepteurs leucine et sérine. Dans le cas du $t$-ARN valine, par contre, les colonnes RPC-fréon (NISHIMURA et WEINSTEIN, I969) se montrent plus sélectives.

5. L'analogie constatée entre les fractionnements des $t$-ARN de levure et de Mammifères d'une part, la similitude entre ceux des $t$-ARN de foie et de glande mammaire d'autre part, permet de penser qu'ils donnent une image représentative de 1'hétérogénéité réelle des ARN de transfert. Les réserves formulées par GiLIAM et al. (1967) restent toutefois justifiées.

6. L'élution précoce d'un $t$-ARN méthionine (tube 52, fig. I ; tube 54, fig. 2) permet d'envisager favorablement sa purification. Ce travail est en cours dans notre laboratoire.

Reçu pour publication en octobre 1969.

\title{
SUMMARY
}

\author{
FRACTIONATION OF MAMMALIAN TRANSFER RIBONUCLEIC ACIDS \\ ON BENZOYL-DIETHYLAMINOETHYL-CELLULOSE
}

In the course of a general survey of fractionation techniques for mammalian transfer ribonucleic acids, benzoyl-DEAE-cellulose was used for seperating the specific transfer RNAs of alanine, arginine, leucine, methionine, serine and valine in rabbit liver and ovine mammary gland.

Each fractionation required approximately ioo mg of crude t-RNA. After chromatography, the specific $t-R N A$ s were isolated by acylation with radioactive amino-acids.

Our results point to the heterogeneity of all investigated t-RNAs. The distribution patterns of the RNAs in the two organs are markedly similar (fig. I and 2).

Consistent similarities with the t-RNAs found in yeast (GILLAM et al., 1967) can be noticed. However, there remains several differences in number and proportion of the various iso-acceptors.

The best purification result (4o per.cent) was obtained with methionine t-RNA.

\section{RÉFÉRENCES BIBLIOGRAPHIQUES}

Brunngraber E. F., I 962 . A simplified procedure for the preparation of " soluble " RNA from rat liver. Biochem. Biophys. Res. Commun., 8, I-8.

Deutscher M. P., I 967 . Rat liver glutamyl ribonucleic acid synthetase. I. Purification and evidence for separate enzymes for glutamic acid and glutamine. J. Biol. Chem., 242, I123-Ir39.

Gillam I., Millward S., Blew D., von Tigerstrom M., Wimmer E., Tener G. M., I967. The separation of soluble ribonucleic acids on benzoylated diethylaminoethyl-cellulose. Biochemistry, $\mathbf{6}$, 3043-3056. 
Hoskinson R. M., Khorana H. G., 1965. Studies on Polynucleotides. XLI. - Purification of phenylalanine specific transfer ribonucleic acid from yeast by countercurrent distribution. $J$. Biol. Chem., 240, $2129-2134$.

Muench K. H., Berg P., ig66. Cantoni G. L., Davies D. R. In Procedures in Nucleic Acid Research, 375-383. A Harper International Edition. Harper and Row, New York, Evanston, London, John Weatherhill, Inc., Tokyo.

Nishimura S., Weinstein I. B., I969. Fractionation of Rat liver transfer ribonucleic acid. Isolation of tyrosine, valine, serine and phenylalanine transfer ribonucleic acids and their coding properties. Biochemistry, 8, 832-842.

PÉtrissant G., I968. Étude de l'acide ribonucléique soluble de glande manmaire de Brebis. I. Préparation, comportement chromatographique, composition nucléotidique. Bull. Soc. Chim. Biol., 50, 47-6I.

Pétrissant G., r969. Étude de l'acide ribonucléique soluble de glande mammaire de Brebis. II. Pou. voir accepteur. Bull. Soc. Chim. Biol., 51, 669-689.

SARIN P. S., ZAMECNIK P. C., rg64. On the stability of aminoacyl-s-RNA to nucleophilic catalysis. Biochim. Biophys. Acta, 91, 653-655. 